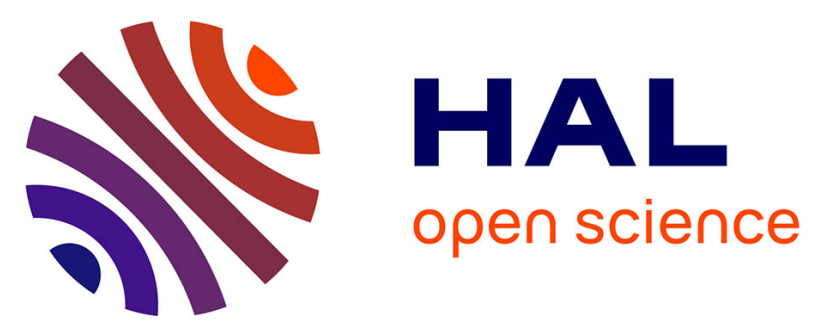

\title{
Neurodevelopmental disorders modify neurodegenerative diseases: a medical history to screen in memory clinic
}

Eloi Magnin, Julie Monnin, Sylvie Chokron, Servane Mouton, Catherine

Thomas Antérion, Sandrine Basaglia-Pappas, Laure Pisella, Isabelle Bernard, Olivier Martinaud, Mathilde Sauvée, et al.

\section{To cite this version:}

Eloi Magnin, Julie Monnin, Sylvie Chokron, Servane Mouton, Catherine Thomas Antérion, et al.. Neurodevelopmental disorders modify neurodegenerative diseases: a medical history to screen in memory clinic. Psychologie \& NeuroPsychiatrie du vieillissement, 2021, 19 (2), pp.181-190. 10.1684/pnv.2021.0936 . hal-03366484

\section{HAL Id: hal-03366484 https://hal.science/hal-03366484}

Submitted on 20 Oct 2021

HAL is a multi-disciplinary open access archive for the deposit and dissemination of scientific research documents, whether they are published or not. The documents may come from teaching and research institutions in France or abroad, or from public or private research centers.
L'archive ouverte pluridisciplinaire HAL, est destinée au dépôt et à la diffusion de documents scientifiques de niveau recherche, publiés ou non, émanant des établissements d'enseignement et de recherche français ou étrangers, des laboratoires publics ou privés. 


\section{Les troubles neurodéveloppementaux miment ou influencent les pathologies neurodégénératives : Prise en compte des antécédents neurodéveloppementaux en consultation mémoire.}

Neurodevelopmental disorders modify neurodegenerative diseases: a medical history to screen in memory clinics.

Eloi Magnin, ${ }^{1,2,3} *$, Julie Monnin ${ }^{1,2,4,5}$, Sylvie Chokron ${ }^{6,7}$, Servane Mouton ${ }^{8}$, Catherine Thomas Antérion $^{9,10}$, Sandrine Basaglia-Pappas ${ }^{11,12}$, Laure Pisella ${ }^{13}$, Isabelle Bernard ${ }^{14}$, Olivier Martinaud ${ }^{15,16}$, Mathilde Sauvée ${ }^{17,18}$, Aurélie Richard-Mornas ${ }^{19,20}$, Ilham Ryff ${ }^{1,4}$, pour la commission du GREDEVad (Groupe de réflexion sur l'évaluation des troubles neurodéveloppementaux de l'adulte) au sein GRECO (Groupe de réflexion sur l'évaluation cognitive).

Affiliation:

${ }^{1}$ Centre Mémoire Ressources et Recherche (CMRR), Centre Expert Parkinson (CEP), Service de Neurologie, CHRU Besançon, F-25000Besançon, France

${ }^{2}$ Neurosciences intégratives et cliniques EA481, Univ. Bourgogne Franche-Comté,F-25000 Besançon, France

${ }^{3}$ Commission GREDEVad (Groupe de Réflexion sur l'Evaluation des troubles neuroDEVeloppmentaux adulte) au sein du GRECO (Groupe de Réflexion sur l'Evaluation Cognitive)

${ }^{4}$ Centre Expert Troubles Bipolaires et Dépression Résistante, Service de Psychiatrie, CHRU Besançon, F-25000Besançon, France

${ }^{5}$ Centre d'Investigation Clinique, CIC INSERM1098, Chu de Besançon.

${ }^{6}$ Institut de Neuropsychologie Neurovision et Neurocognition, Hôpital Fondation Rothschild, Paris, France. 
${ }^{7}$ Université de Paris CNRS Integrative Neuroscience and Cognition Center Paris France, Paris, France.

${ }^{8}$ Cabinet de neurologie libérale, Neuville Sur Saone, France.

${ }^{9}$ Laboratoire EMC, EA3082, Université Lyon, Bron cedex, France

${ }^{10}$ Cabinet de neurologie libérale, Lyon, France.

11 Service de neurologie et neuropsychologie, CHU Saint-Etienne, France.

${ }^{12}$ Service de psychologie cognitive et neuropsychologie, Université de Mons, Belgique.

${ }^{13}$ Centre de Recherche en Neurosciences de Lyon (CRNL), Université de Lyon, Bron, France.

${ }^{14}$ Cabinet de neurologie libérale, Cholet, France.

${ }^{15}$ Service de neurologie, CHU, Caen, France.

${ }^{16}$ Neuropsychologie et Imagerie de la mémoire humaine, Inserm U1077, pôle des formations et de recherche en santé, université de Caen-Normandie, Caen, France.

${ }^{17}$ Centre Mémoire de Ressources et de Recherche, Pôle de Psychiatrie et Neurologie, CHU, Grenoble, France.

${ }^{18}$ Université Grenoble Alpes, Université Savoie Mont Blanc, CNRS UMR 5105, Laboratoire de Psychologie et Neurocognition (LPNC), Grenoble, France

19 Service de neurologie fonctionnelle et épileptologie, Hospices Civils de Lyon, Bron France.

${ }^{20}$ Centre de Rechenerche en Neurosciences de Lyon, INSERM U1028, CNRS UMR5292, Université Lyon 1, Lyon, France.

*Auteur correspondant : 
Eloi Magnin, service de Neurologie, CHU, Boulevard Fleming, 25000 Besançon, France. Tel.: +33 381668098; Fax: +33 381668740; E-mail: eloi.magnin@ univ-fcomte.fr 


\section{RÉSUMÉ}

Les troubles neurodéveloppementaux sont fréquents en population générale et perdurent souvent tout au long de la vie même s'ils peuvent être masqués par des mécanismes de compensation. Ces troubles sont pourtant souvent méconnus ou sous-estimés chez le sujet adulte et ce, même lorsqu'ils ont été diagnostiqués pendant l'enfance. Ils peuvent aussi interagir de manière complexe avec les processus neurodégénératifs, modifiant ainsi les présentations habituelles de ces pathologies. Prendre en compte cette dimension neurodéveloppementale au cours des consultations mémoire permet de mieux appréhender les différentes trajectoires cognitives tout au long de la vie. Cette nouvelle dimension vient enrichir l'idée d'une démarche intégrative en adoptant une approche médicale cognitivocomportementale de précision qui se veut personnalisée.

Mots clés : Troubles neurodéveloppementaux; maladie neurodégénérative ; démence atypique, adulte. 


\begin{abstract}
Neurodevelopmental disorders are frequent in the general population and are often lifelong conditions despite sometimes being masked by compensation mechanisms. These conditions are often unknown or underestimated in adults, even when diagnosed in childhood. Neurodevelopmental disorders share similarities with and frequently interact in a complex way with neurodegenerative disorders. Considering these aspects during memory clinic assessments can provide a new perspective on lifelong neurocognitive trajectories. Assessing both neurodevelopmental and neurodegenerative dimensions is challenging but should improve diagnostic accuracy. It is therefore necessary to understand the lifelong specific neurocognitive trajectory of each patient in order to develop personalized and focused cognitive medicine and care.
\end{abstract}

Keywords: Neurodevelopmental disorders; neurodegenerative disease; atypical dementia 


\section{POINTS CLES}

- Les troubles neurodéveloppementaux chez l'adulte sont fréquents, souvent méconnus et leur impact au quotidien est souvent sous-estimé.

- Les troubles neurodéveloppementaux perdurent souvent tout au long de la vie mais peuvent être masqués par des mécanismes de compensations.

- Les troubles neurodéveloppementaux modifient l'expression des pathologies neurodégénératives

- Leur prise en compte en consultation mémoire est complexe mais permet d'affiner les diagnostics et d'adapter les prises en charge. 


\section{Introduction}

Les troubles neurodéveloppementaux constituent un groupe de syndromes définis dans le DSM-V qui se manifestent tôt au cours de l'enfance par un développement cérébral atypique amenant à des fonctionnements différents ou des dysfonctions. Cela peut entrainer des troubles cognitifs et/ou comportementaux pouvant retentir sur différents champs de la vie quotidienne (familial, social, scolaire, professionnel) [1]. Les troubles neurodéveloppementaux sont très fréquents en population générale avec une prévalence évaluée à $15 \%$ dans certains pays [2], ce qui en fait un enjeu de santé publique, restant cependant peu connu des médecins s'occupant d'adultes ou de personnes âgées. Plusieurs présentations cliniques sont décrites telles que les troubles spécifiques des apprentissages (dyslexie ou dyscalculie par exemple), le trouble déficitaire de l'attention avec ou sans hyperactivité (TDAH), le trouble développemental du langage (anciennement appelé dysphasie développementale), le trouble de développement de la coordination (anciennement appelé dyspraxie développementale) et le trouble du spectre de l'autisme. Plusieurs d'entre eux peuvent s'associer et le terme de "'multidys" est de plus en plus utilisé pour rendre compte de ces différentes co-morbidités.

Souvent considérés comme des troubles relevant de la pédiatrie et/ou de la pédopsychiatrie, avec simplement un retard dans le développement qui se comblera avec le temps, cette condition cognitive et ce fonctionnement neurologique perdurent pourtant souvent tout au long de la vie, avec simplement des mécanismes de compensation ou d'adaptation plus ou moins coûteux en terme de surcharge cognitive. Au quotidien par exemple, des sujets adultes dyslexiques, au cours de lectures, ou des sujets adultes présentant un TDAH, au cours de remplissages de formulaires administratifs, pourront présenter un ralentissement en raison d'une stratégie de rétrocontrôle par vérification pour limiter leurs erreurs. [3-5]. Ainsi face à 
un évènement de vie (comme une naissance ou un deuil) ou évènement de santé (trouble du sommeil, troubles métaboliques), retentissant sur les ressources cognitives, ces mécanismes peuvent être décompensé et les symptômes et gènes au quotidien peuvent se manifester de manière plus importante pouvant mimer une pathologie évolutive neurodégénérative.

C'est pourquoi ces patients, dont l'antécédent neurodeveloppemental est souvent méconnus chez l'adulte, peuvent présenter au cours des évaluations en consultation mémoire adulte des profils cognitifs atypiques constitutionnels qui n'impliquent pas pour autant une pathologie neurodégénérative. Ces trajectoires cognitives différentes peuvent aussi perturber les trajectoires habituelles du vieillissement normal et, le cas échéant, d'authentiques processus neurodégénératifs, modifiant ainsi leur présentation clinique [6-7]. De nombreuses hypothèses quant aux interactions possibles entre ces 2 situations aux deux extrêmes de la vie existent mais ne sont pas encore élucidées (figure 1). Le défi sera pour les cliniciens d'être capables d'avoir une représentation cognitive globale de la personne et d'intégrer à leur démarche clinique ce fonctionnement cognitif différent afin de pouvoir évoquer ce diagnostic différentiel ou cette comorbidité au cours de la démarche diagnostique [8].

Cette dimension neurodéveloppementale au sein des pathologies neurodégénératives commence à être étudiée et décrite dans la littérature (for a review see [9]). L’objectif de cette revue est de décrire les associations entre troubles neurodéveloppementaux et maladies neurodégénératives. Cette approche poursuit tout d'abord un but fondamental, celui de comprendre le lien fonctionnel et neuro-anatomique entre ces deux entités. D'autre part, cette démarche vise un but clinique à long terme, en consultations mémoire adulte, celui de sensibiliser les professionnels de santé quant à l'importance du repérage des troubles neurodéveloppementaux à tout âge [10]. 


\section{Impact neurodéveloppemental sur l'expression clinique des pathologies neurodégénératives}

La prévalence des formes atypiques non amnésiques au sein de la maladie d'Alzheimer est d'environ $15 \%$ [11], elle est similaire à celle des troubles neurodéveloppementaux en population générale [2] ceci soulève l'hypothèse que les troubles neurodéveloppementaux pourraient être un facteur de risque de développement d'une présentation atypique de pathologie neurodégénérative [7]. En effet, avoir un possible antécédent de trouble neurodéveloppemental est plus souvent observé (Odd Ratio $=13.1 ; 95 \%$ IC 1.3-128.4) chez les patients ayant des présentations cliniques atypiques et focales de maladie d'Alzheimer comme une atrophie corticale postérieure ou une aphasie progressive primaire ( 7 patients sur 17, i.e. $41 \%$ ) que chez les patients présentant des formes typiques amnésiques ( 8 patients sur 68 , i.e. $11 \%, p=0.02$ ) [7]. Il est possible que le fait d'avoir constitutionnellement un profil cognitif neurodéveloppemental atypique, avec une structuration différente des réseaux neuronaux (anomalies de développement corticale, connectivité différente), puisse conduire à des processus neurodégénératifs également atypiques.

Cependant, même en tenant compte de la fréquente méconnaissance des troubles neurodéveloppementaux, tous les patients présentant des formes atypiques ne semblent pas forcément présenter d'antécédent de ce type ce qui suggère des interactions complexes et probablement multifactorielles dans ce cadre. Les mécanismes sous-tendant ces différents processus en terme de causalité, de facteurs de risques, de facteur de protection de comorbidités, restant encore largement méconnus, les relations sont souvent descriptives et/ou hypothétiques (figure 1). 
Les différentes formes cliniques atypiques des pathologies neurodégénératives seraient associées de manière préférentielle avec certains syndromes neurodéveloppementaux, en lien avec leurs points communs cognitifs et structurels.

\section{Aphasie progressive primaire}

Marcel Mesulam a évoqué, dès les années 90, une possible association entre les aphasies progressives primaires et la dyslexie développementale, la considérant comme un facteur de risque possible [12]. Les premiers éléments en faveur de cette interaction sont rapportées seulement 20 ans plus tard et concernent le sous-type logopénique d'aphasie progressive primaire pour lequel un antécédent de dyslexie développementale est retrouvé deux fois plus souvent que dans la population générale [13]. Le point commun identifié dans les deux entités est le déficit cognitif similaire qui touche la mémoire à court terme auditivo-verbale, et plus spécifiquement, la boucle phonologique. De plus, les dysfonctionnements structuraux associés à ces deux troubles sont souvent observés dans le même territoire anatomique : le carrefour pariétotemporal gauche. C'est le cas des anomalies corticales de développement pour la dyslexie, et de l'atrophie avec accumulation de lésions neuropathologiques de pathologie neurodégénérative (le plus souvent de maladie d'Alzheimer biologique) pour l'aphasie progressive primaire logopénique, Dans cette étude [13], l'atrophie hémisphérique gauche des portions postérieures des gyri temporaux moyens et supérieurs était moindre chez les patients présentant une aphasie progressive primaire ayant eu une dyslexie développementale en comparaison à ceux sans trouble neurodéveloppemental. Les patients dyslexiques avaient déclaré des symptômes plus précocement (en moyenne 6.2 ans) mais la sévérité de leurs troubles au MMSE et aux tâches de dénomination était moindre que pour les patients sans trouble neurodéveloppemental. Ces interactions complexes peuvent suggérer que les patients dyslexiques seraient symptomatiques avec une moindre charge lésionnelle neurodégénérative (i.e. moindre atrophie) et présenteraient donc une moindre réserve 
cognitive focalisée à un domaine cognitif spécifique (i.e. au niveau de la boucle phonologique) constituant ainsi un «point faible» ou une fragilité d'origine neurodéveloppementale avec une addition des déficits constitutionnels et acquis. Ils présentent cependant également plus de mécanismes de compensation, acquis tout au long de la vie par eux-même ou grâce à une prise en charge orthophonique ou neuropsychologique dans l'enfance par exemple (i.e. développant ainsi une autre composante de la réserve cognitive). Ce «fardeau» compensatoire permanent pourrait expliquer la précocité des troubles cliniques par un phénomène de consommation des ressources cognitives ou surcharge cognitive alors que les patients non dyslexiques sont encore asymptomatiques. Cependant, ces mécanismes compensatoires deviennent un atout secondairement pour faire face à la pathologie, limitant l'impact de ce processus neurodégénératif sur leur fonctionnement cognitif aux évaluations neuropsychologiques (i.e. moindre déficit cognitif). Dans une autre étude [14], l'exploration neuropathologique de trois patients aux antécédents de dyslexie ayant eu une aphasie progressive primaire logopénique montre l'association dans la région périsylvienne de dysplasies corticales focales (i.e. delaminations corticales, neurones géants, immatures, dysplasiques, hétérotopiques ou en surnombre dans les différentes couches corticales) avec une concentration plus importante de lésions de maladie d'Alzheimer. Ces résultats ajoutent à l'hypothèse du «point faible » ou de la fragilité d'origine neurodéveloppementale, la possibilité d'un emballement neurodégénératif localisé au niveau de la zone d'anomalie développementale.

Outre les altérations cérébrales structurales communes, le contexte génétique pourrait participer à ces associations, puisqu'une surreprésentation de troubles du langage développementaux et de fragilité du réseau neuronal du langage hémisphérique gauche, associés à un moindre volume et une moindre connectivité au repos, est rapportée dans les familles de patients ayant une aphasie progressive primaire [15]. 
Certains gènes de susceptibilité impliqués dans la dyslexie développementale (KIAA0319 et CNTNAP2) sont d'ailleurs associés à une atrophie hémisphérique gauche plus importante chez les patients ayant une dégénérescence lobaire fronto-temporale [16-17]. Cependant, dans ces études, la présentation initiale des patients ayant ces gènes n'incluait pas de manière plus fréquente une aphasie progressive primaire (c'est-à-dire une forme langagière de dégénérescence fronto-temporale) que des formes comportementales.

Enfin, la dyslatéralité développementale (i.e. absence de préférence manuelle droite type gaucherie ou ambidextrie), un autre fonctionnement développemental atypique ne pouvant pourtant pas être considéré réellement comme un trouble car peu handicapant au quotidien, est souvent associé aux dyslexies développementales [18]. Cet antécédent est aussi surreprésenté dans les formes sémantiques d'aphasie progressive primaire [13]. Ce lien n'est pas clairement élucidé. Du point de vue structural, la modification du réseau neuronal du langage avec une moindre spécificité hémisphérique gauche chez ces patients mal latéralisés peut participer à la fragilisation des fonctions langagières incluant la mémoire sémantique. La perte d'asymétrie du planum temporale et de la pars triangularis, rapportée chez les sujets mal latéralisés, est ainsi associée à de moindres performances verbales [19].

\section{Maladie de Parkinson et Maladie à corps de Lewy}

Un possible fonctionnement prémorbide évoquant un TDAH, évalué par l'auto-questionnaire de la WURS, qui explore la sévérité des symptômes du TDAH avec des échelles de Likert de 0 (« pas du tout ou légèrement présent ») à 4 («beaucoup »), est rapporté presque 3 fois plus souvent chez des patients présentant une maladie à corps de Lewy (48\% des 109 patients) que chez des patients avec une maladie d'Alzheimer ou chez des sujets contrôles (environ 15\% pour les deux groupes) [6]. De même, sans pour autant dépasser les seuils diagnostiques des 
échelles diagnostiques, des symptômes d'inattention et d'hyperactivité dans l'enfance sont aussi modérément plus important chez les patients présentant une maladie de Parkinson par rapport à des sujets contrôles, (score à la WURS $14.4+/-13$ vs 9.8+/-10; $\mathrm{p}=0.01$, valeur seuil > 30) [20]. Les points communs entre TDAH et ces deux types de synucléinopathie sont cliniques et physiopathologiques. D'une part, ils partagent des symptômes cognitifs tels que les troubles attentionnels et le syndrome dysexécutif [21]. D’autre part, le vaste réseau attentionnel fronto-pariétal [22] et le dysfonctionnement dopaminergique au sein des circuits fronto-striato-cerebelleux participant au contrôle cognitif [23-24] sont impliqués à la fois dans le TDAH et dans ces pathologies neurodégénératives.

\section{Atrophie corticale postérieure}

Les atrophies corticales postérieures sont fréquemment associées à des troubles neurodéveloppementaux [25]: d'une part les troubles des apprentissages mathématiques, et d'autre part les troubles neurovisuels de l'enfance. Les symptômes neurovisuels et la dyscalculie sont des dysfonctions cognitives communes à ces entités [26-27]. Les troubles neurovisuels de l'enfance, survenant au cours du développement et pouvant mimer une déficience intellectuelle et/ou des signes autistiques correspondent, anatomiquement, souvent à une dysfonction cérébrale postérieure en particulier des régions occipitales et/ou pariétales. Les troubles des apprentissages mathématiques, quant à eux, correspondent souvent à un dysfonctionnement des régions pariétales [28-29]. Ces régions occipito-pariétales sont également les zones de neurodégénérescence impliquées dans les atrophies corticales postérieures [27]. Une étude portant sur 95 patients présentant une atrophie corticale postérieure a montré une surreprésentation d'antécédents neurodéveloppementaux comparativement à des patients atteints de maladie d'Alzheimer de présentation typique amnésique $(13.6 \%$ versus $1 \%$; $p$ <0.001) [25]. Les patients présentant une atrophie corticale postérieure avec un trouble neurodéveloppemental avaient également une atrophie plus 
asymétrique et prédominante à droite en comparaison à des patients sans antécédent neurodéveloppemental [25]. Comme pour les aphasies progressives primaires, les patients avec un antécédent neurodéveloppemental présentaient des performances cognitives meilleures que les patients sans antécédent neurodéveloppemental au MMSE, fluences sémantiques et empans de chiffres envers, suggérant une moindre diffusion des lésions ou des mécanismes de compensations déjà en place limitant les déficits.

Un antécédent de TDAH a été rapporté avec un cas d'atrophie corticale postérieure questionnant sur un lien possible entre ces deux syndromes [7]. Les troubles attentionnels visuels sont des symptômes communs aux deux entités [24, 27], tout comme, d'un point de vue structurel, les anomalies du lobe pariétal et la dysrégulation des noyaux gris centraux incluant notamment le thalamus, un relais visuel important [24-27]. On soulignera également que la maladie à corps de Lewy peut se présenter initialement comme une atrophie corticale postérieure [27] ce qui supporte l'existence d'un lien entre ce syndrome, l'étiologie sousjacente parfois à son origine, comme la maladie à corps de Lewy, et le TDAH.

Une étude pangénomique exploratoire chez 293 patients avec atrophie corticale postérieure suggère l'implication de trois gènes dont deux (SEMA3C et CNTNAP5) qui pourraient contribuer également à des processus en lien avec le développement cérébral [30]. SEMA3C est impliqué dans le développement cortical et hippocampique, ainsi que la maturation du système visuel et CNTNAP5 est associé avec le trouble du spectre de l'autisme, ayant des interactions complexes avec les troubles neurovisuels de l'enfant [26], et la maladie bipolaire, pathologie psychiatrique ayant aussi une forte composante neurodéveloppementale et des lien complexe avec les pathologies neurodégénératives [31]. 


\section{Diagnostic différentiel : des troubles neurodéveloppementaux méconnus peuvent mimer des syndromes neurodégénératifs au cours du vieillissement}

Les troubles neurodéveloppementaux ne sont pas toujours diagnostiqués dans l'enfance [32] et par la suite à l'âge adulte, d'autant plus chez les patients les plus âgés pour qui ces diagnostics n'étaient pas autant explorés à l'époque de leur enfance qu'actuellement [8]. De plus, même si un trouble neurodéveloppemental a été évoqué dans l'enfance, il n'est souvent pas rapporté spontanément par les patients dans leurs antécédents car il est considéré comme un problème scolaire passé plutôt qu'une histoire médicale pouvant encore avoir des conséquences, notamment sur une évaluation cognitive en consultation mémoire [8-9].

Et pourtant, même s'il peut être compensé, le fonctionnement cognitif atypique constitutionnel des patients avec un trouble neurodéveloppemental perdure tout au long de la vie. Au cours du vieillissement, habituellement associé à des troubles attentionnels légers, un discret syndrome dysexécutif et un ralentissement dans le traitement de l'information, le fonctionnement cognitif de ces patients peut parfois se modifier, en engendrant une moindre efficacité des mécanismes de compensation par un phénomène de surcharge ou d'épuisement des ressources cognitives [33]. Des facteurs généraux influençant la cognition peuvent s'ajouter et ainsi consommer une partie des ressources cognitives, notamment en population gériatrique fragile, tels qu'un syndrome d'apnée du sommeil, de la iatrogénie, des troubles de l'humeur ou encore des troubles endocriniens [9]. Une plainte cognitive peut ainsi s'installer et amener les patients à consulter en centre mémoire. Si leur fonctionnement cognitif atypique de base est méconnu, le profil cognitif présentant des déficits constitutionnels par rapport à la norme peut être considéré, à tort, comme un déclin récent et ainsi les classer dans la population à risque de développer une maladie neurodégénérative selon le DSM-V [8-9, 21, 34]. En fonction des troubles cognitifs et/ou comportementaux observés d'origine neurodéveloppementale méconnue, il est possible de conclure à un trouble neurocognitif 
léger exécutif, amnésique ou multidomaine ou encore à une aphasie progressive primaire par exemple $[9,21]$. Bien entendu, la recherche systématique d'une cause neurodégénérative surajoutée reste nécessaire avant de considérer que ces profils sont expliqués par un trouble neurodéveloppemental isolé [35].

En cas de présentations initialement considérées comme des troubles neurocognitifs légers, des aphasies progressives primaires, des atrophies corticales postérieures ou des dégénérescences lobaires fronto-temporales qui se révèlent non-évolutives au cours d'un suivi clinique et neuropsychologique à long terme, le diagnostic de pathologie neurodégénérative suspectée doit être remis en question et, un trouble neurodéveloppemental sous-jacent devrait être systématiquement recherché. Les phénocopies de forme comportementale de dégénérescence fronto-temporale illustrent bien cette situation. Il s'agit de présentations cliniques sans anomalie d'imagerie associée, n'évoluant pas ou très peu dans le temps au cours du suivi et pour lesquelles l'étiologie sous-jacente reste incertaine. Outre des pathologies neurodégénératives très lentement évolutives ou des pathologies psychiatriques d'apparition tardive, des troubles neurodéveloppementaux méconnus tels que le TDAH ou le trouble du spectre de l'autisme ont été associés à ces présentations atypiques [36].

Evoquer un trouble neurodéveloppemental de l'adulte est important pour faire le diagnostic différentiel mais également identifier ces éventuelles comorbidités associées à des pathologies neurodégénératives (figure 2) risquant d'avoir plus souvent des présentations atypiques et ce, dans le but d'affiner les diagnostics et les prises en charge personnalisées. Ceci représente un véritable défi en raison notamment de la nécessité d'une anamnèse très exhaustive, d'un suivi clinique et neuropsychologique à long terme qui se révèle être des outils diagnostiques majeurs pour ces situations complexes [8]. 


\section{Diagnostiquer un trouble neurodéveloppemental au cours du vieillissement normal et/ou son éventuelle association à une authentique pathologie neurodégénérative?}

Le diagnostic de certitude pour les pathologies neurodégénératives et pour les troubles neurodéveloppementaux est post-mortem, à l'examen neuropathologique, ou génétique, avec l'identification de mutation causale de la symptomatologie [37]. Dans la majorité des situations in vivo, le diagnostic n'est que probabiliste à partir de classifications clinicoradiologiques permettant parfois d'évaluer le risque «possible» et «probable » d'avoir ce diagnostic, en fonction du nombre de critères présentés par les patients. Très peu de biomarqueurs biologiques ou d'imagerie fiables existent pour améliorer cette acuité diagnostique, en dehors de ceux développés pour la maladie d'Alzheimer (imagerie TEP amyloïde, imagerie TEP Tau, dosage des protéines Tau et amyloïdes dans le liquide cérébrospinal, génotype $A P O e$ ) qui ne sont pas toujours applicables à la pratique clinique quotidienne.

La confusion diagnostique dans les pathologies neurodégénératives est fréquente, à la fois entre les différentes étiologies neurodégénératives et syndromes cliniques mais aussi avec des diagnostics différentiels tels que des pathologies psychiatriques ou des troubles neurodéveloppementaux méconnus (figure 2). C'est un problème majeur des consultations mémoire avec un taux d'erreur pour le diagnostic de maladie d'Alzheimer évalué entre 20 et 30\%. Il se retrouve dans le cadre de l'activité clinique habituelle, et peut avoir des conséquences délétères par excès ou par défaut sur les prises en charge médicamenteuses et médicosociales de ces patients. Il est également présent dans le cadre de la recherche clinique avec de possibles inclusions à tort pouvant induire une perte de puissance statistique avec un «bruit de fond » pouvant mettre en péril certaines découvertes, notamment thérapeutiques [38-42]. Les troubles neurodéveloppementaux peuvent faire partie des facteurs confondants 
et/ou des diagnostics différentiels participant à certaines de ces erreurs diagnostiques [8, 4344]. Ainsi, la précision diagnostique des évaluations cognitives de l'adulte et de la personne âgée pourrait être améliorée par un repérage systématique des troubles neurodéveloppementaux et de leur impact sur les profils clinique et cognitif des patients. Connaître les trajectoires neurocognitives des patients tout au long de leur vie permettrait alors d'avoir une médecine de précision personnalisée dans le domaine neurocognitif [8-9]. Le rôle probablement complexe des troubles neurodéveloppementaux dans le mode évolutif rapide ou lent, et/ou l'apparition précoce ou tardive des processus neurodégénératifs, nécessitera la mise en place de grandes cohortes prospectives longitudinales sur de longues périodes de suivi.

Poser un diagnostic de trouble neurodéveloppemental chez un adulte, et encore plus chez une personne âgée, repose sur une évaluation anamnestique a posteriori de symptômes présents depuis l'enfance (par exemple des retards dans les acquisitions du langage ou de la marche, des redoublements en primaire) et des facteurs de risque au cours du développement précoce (par exemple des troubles périnataux ou des antécédents familiaux de troubles neurodéveloppementaux). L'absence d'informations extérieures sur cette période de vie (parents, fratrie, carnet de santé, bulletins scolaires) est fréquente, surtout en population gériatrique, et vient compliquer cette démarche diagnostique chez des patients consultant pour des plaintes cognitives, potentiellement moins informatifs ou anosognosiques. Cela nécessite d'explorer systématiquement la période développementale par un repérage et de compléter ensuite par une investigation exhaustive comprenant l'utilisation de questionnaires et de trames d'anamnèse semi-structurée traduits en français et validés pour cette population, comme l'échelle de la WURS [45] et l'anamnèse semi-structurée de DIVA [46] pour une évaluation d'un TDAH de l'adulte, le Quotient du spectre de l'autisme (QA) [47] ou un 
questionnaire de dépistage court tel que le 30-Item Self-Report Questionnaire évaluant les difficultés en langage, mathématique, attention et fonction exécutive [10], et d'adjoindre a ces explorations, une évaluation neuropsychologique à la recherche de troubles cognitifs spécifiques soutenant ces hypothèses. Actuellement les outils sont rares et peu traduits en français, n'explorent pas de tous les troubles neurodeveloppementaux, et leur validation est parfois peu robuste, surtout chez l'adulte et d'autant plus chez la personne âgée ou la personne ayant une pathologie neurodégénérative surajoutée [8]. De plus, il n'existe pas de consensus quant aux tests neuropsychologiques à utiliser chez cette population adulte.

Comme cela a déjà été évoqué, une anamnèse très détaillée et, dans la mesure du possible, une hétéroanamnèse fiable auprès de proches informatifs sur la période de l'enfance, le suivi à long terme clinique et neuropsychologique, constituent des points clefs pour identifier ces situations [8]. Une stabilité à long terme du profil cognitif avec une anamnèse évocatrice d'un trouble neurodéveloppemental suggèrent ainsi que celui-ci est relativement isolé, alors qu'un déclin progressif des performances cognitives semble plus évocateur d'une composante neurodégénérative surajoutée [48].

En conclusion, il existe des interactions complexes entre les troubles neurodéveloppementaux et les pathologies neurodégénératives mais ces résultats reposent encore sur de petites séries de patients, nécessitant confirmation sur de plus grandes cohortes, avec des outils et procédures diagnostiques pour repérer les troubles neurodéveloppementaux nécessitant d'être validés et harmonisés [7-10]. Le diagnostic de trouble neurodéveloppemental est aujourd'hui uniquement basé sur la clinique et l'évaluation cognitive avec des outils et des stratégies à améliorer pour cette population. Une vision pluridisciplinaire et pluri-professionnelle est fréquemment nécessaire au cours de la démarche diagnostique avec notamment l'appui de psychiatres et/ou psychologues de l'adulte formés aux diagnostics de TDAH et/ou de TSA et sensibilisés aux autres troubles neurodéveloppementaux mais aussi pour identifier d'éventuels 
diagnostics différentiels ou comorbides (comme par exemple des troubles de personnalité, un trouble du spectre de la schizophrénie ou encore un trouble de l'humeur). Le développement de biomarqueurs d'imagerie ou biologiques est nécessaire pour améliorer la précision et le degré de confiance de ces diagnostics [49-50]. En combinaison avec les biomarqueurs des maladies neurodégénératives, les doubles diagnostics pourraient aussi être confortés et posés plus précocement sans nécessité d'un long suivi dans l'attente d'un déclin. 
Liens d'intérêts : Les auteurs déclarent ne pas avoir de lien d'intérêt en rapport avec cet article.

\section{Références}

1. American Psychiatric Association. Diagnostic and statistical manual of mental disorders (5th ed.). Washington, DC : American Psychiatric Association, 2013.

2. Dietrich KN, Eskenazi B, Schantz S, Yolton K, Rauh VA, Johnson CB, et al. Principles and practices of neurodevelopmental assessment in children: lessons learned from the Centers for Children's Environmental Health and Disease Prevention Research. Environ Health Perspect $2005 ; 113: 1437-46$.

3. Peterson RL, Pennington BF, Olson RK, Wadsworth S. Longitudinal Stability of Phonological and Surface Subtypes of Developmental Dyslexia. Sci Stud Read 2014 ; 18 : $347-62$.

4. Kessler RC, Green JG, Adler LA, Barkley RA, Chatterji S, Faraone SV, et al. Structure and diagnosis of adult attention-deficit/hyperactivity disorder: analysis of expanded symptom criteria from the Adult ADHD Clinical Diagnostic Scale. Arch Gen Psychiatry $2010 ; 67: 1168-78$.

5. Chokron S, Demonet JF. Approche neuropsychologique des troubles des apprentissages. Paris : Ed De Boeck-Solal. 2010.

6. Golimstok A, Rojas JI, Romano M, Zurru MC, Doctorovich D, Cristiano E. Previous adult attention-deficit and hyperactivity disorder symptoms and risk of dementia with Lewy bodies: a case-control study. Eur J Neurol 2011;18:78-84. 
7. Seifan A, Assuras S, Huey ED, Mez J, Tsapanou A, Caccappolo E. Childhood Learning Disabilities and Atypical Dementia: A Retrospective Chart Review. PLoS One 2015;10:e0129919.

8. Magnin E, Maurs C. Attention-deficit/hyperactivity disorder during adulthood. Rev Neurol (Paris) 2017;173:506-15.

9. Magnin E. Neurodevelopmental and neurodegenerative similarities and interactions: a point of view about lifelong neurocognitive trajectories. J Alz Dis 2021;79:1397-407.

10. Seifan A, Shih C, Hackett K, Pensack MJ, Schelke MW, Lin M, et al. Detection of neurodevelopmental diversity in memory clinics-Validation of a self-report measure. Res Dev Disabil 2018;77:60-7.

11. Whitwell JL, Dickson DW, Murray ME, Weigand SD, Tosakulwong N, Senjem ML, et al. Neuroimaging correlates of pathologically defined subtypes of Alzheimer's disease: a case-control study. Lancet Neurol 2012; 11:868-77.

12. Mesulam MM, Weintraub S. Spectrum of primary progressive aphasia. In: Rossor MN, ed. Unusual Dementias. London, England: Baillière Tindall; 1992:583-609.

13. Miller ZA, Mandelli ML, Rankin KP, Henry ML, Babiak MC, Frazier DT, et al. Handedness and language learning disability differentially distribute in progressive aphasia variants. Brain 2013;136:3461-73.

14. Miller ZA, Spina S, Pakvasa M, Rosenberg L, Watson C, Mandelli ML, et al. Cortical developmental abnormalities in logopenic variant primary progressive aphasia with dyslexia. Brain Communications 2019; 1:fcz027

15. Weintraub S, Rader B, Coventry C, Sridhar J, Wood J, Guillaume KA, et al. Familial language network vulnerability in primary progressive aphasia. Neurology 2020;95:e847e855. 
16. Paternicó D, Manes M, Premi E, Cosseddu M, Gazzina S, Alberici A, et al. Frontotemporal dementia and language networks: cortical thickness reduction is driven by dyslexia susceptibility genes. Sci Rep 2016;6:30848.

17. Paternicó D, Premi E, Alberici A, Archetti S, Bonomi E, Gualeni V, et al. Dyslexia susceptibility genes influence brain atrophy in frontotemporal dementia. Neurol Genet 2015;1:e24.

18. Eglinton E, Annett M. Handedness and dyslexia: a meta-analysis. Percept Mot Skills 1994;79:1611-6.

19. Nair S, Nenert RE, Allendorfer JB, Goodman AM, Vannest J, Mirman D, et al. Sex, Age, and Handedness Modulate the Neural Correlates of Active Learning. Front Neurosci 2019;13:961.

20. Walitza S, Melfsen S, Herhaus G, Scheuerpflug P, Warnke A, Müller T, et al. Association of Parkinson's disease with symptoms of attention deficit hyperactivity disorder in childhood. J Neural Transm Suppl 2007; 72:311-5.

21. Callahan BL, Bierstone D, Stuss DT, Black SE. Adult ADHD: Risk Factor for Dementia or Phenotypic Mimic? Front Aging Neurosci 2017;9:260.

22. Lin HY, Tseng WY, Lai MC, Matsuo K, Gau SS. Altered resting-state frontoparietal control network in children with attention-deficit/hyperactivity disorder. J Int Neuropsychol Soc 2015;21:271-84

23. Cortese S. The neurobiology and genetics of Attention-Deficit/Hyperactivity Disorder (ADHD): what every clinician should know. Eur J Paediatr Neurol 2012;16:422-33.

24. Redondo B, Molina R, Cano-Rodríguez A, Vera J, García JA, Muñoz-Hoyos A, et al. Visual Perceptual Skills in Attention-deficit/Hyperactivity Disorder Children: The Mediating Role of Comorbidities. Optom Vis Sci 2019;96:655-63. 
25. Miller ZA, Rosenberg L, Santos-Santos MA, Stephens M, Allen IE, Hubbard HI, et al. Prevalence of Mathematical and Visuospatial Learning Disabilities in Patients With Posterior Cortical Atrophy. JAMA Neurol 2018;75:728-37.

26. Chokron S, Kovarski K, Zalla T, Dutton GN. The inter-relationships between cerebral visual impairment, autism and intellectual disability. Neurosci Biobehav Rev 2020;114:20110.

27-. Crutch SJ, Schott JM, Rabinovici GD, Murray M, Snowden JS, van der Flier WM, et al. Consensus classification of posterior cortical atrophy. Alzheimers Dement 2017;13:87084.

28. Molko N, Cachia A, Rivière D, Mangin JF, Bruandet M, Le Bihan D, et al. Functional and structural alterations of the intraparietal sulcus in a developmental dyscalculia of genetic origin. Neuron 2003;40:847-58.

29. Lehman SS. Cortical visual impairment in children: identification, evaluation and diagnosis. Curr Opin Ophthalmol 2012;23:384-7.

30. Schott JM, Crutch SJ, Carrasquillo MM, Uphill J, Shakespeare TJ, Ryan NS, et al. Genetic risk factors for the posterior cortical atrophy variant of Alzheimer's disease. Alzheimers Dement 2016;12:862-71.

31. Masouy A, Chopard G, Vandel P, Magnin E, Rumbach L, Sechter D, et al. Bipolar disorder and dementia: where is the link? Psychogeriatrics 2011;11:60-7.

32. Barbiero C, Lonciari I, Montico M, Monasta L, Penge R, Vio C, et al.The submerged dyslexia iceberg: how many school children are not diagnosed? Results from an Italian study. PLoS One 2012;7:e48082.

33. Laasonen M, Lahti-Nuuttila P, Virsu V. Developmentally impaired processing speed decreases more than normally with age. Neuroreport $2002 ; 13: 1111-3$. 
34. Ivanchak N, Fletcher K, Jicha GA. Attention-deficit/hyperactivity disorder in older adults: prevalence and possible connections to mild cognitive impairment. Curr Psychiatry Rep $2012 ; 14: 552-60$.

35. Metzler-Baddeley C, Salter A, Jones RW. The significance of dyslexia screening for the assessment of dementia in older people. Int J Geriatr Psychiatry $2008 ; 23$ : 766-8.

36. Valente ES, Caramelli P, Gambogi LB, Mariano LI, Guimarães HC, Teixeira AL, et al. Phenocopy syndrome of behavioral variant frontotemporal dementia: a systematic review. Alzheimers Res Ther 2019;11:30.

37. McKhann GM, Knopman DS, Chertkow H, Hyman BT, Jack CR Jr, Kawas CH, et al. The diagnosis of dementia due to Alzheimer's disease: recommendations from the National Institute on Aging-Alzheimer's Association workgroups on diagnostic guidelines for Alzheimer's disease. Alzheimers Dement 2011;7:263-9.

38. Hogervorst E, Bandelow S, Combrinck M, Irani SR, Smith AD. The validity and reliability of 6 sets of clinical criteria to classify Alzheimer's disease and vascular dementia in cases confirmed post-mortem: added value of a decision treeapproach. Dement Geriatr Cogn Disord 2003;16:170-80.

39. Knopman DS, DeKosky ST, Cummings JL, Chui H, Corey-Bloom J, et al. Practice parameter: diagnosis of dementia (an evidence-based review). Reportof the Quality Standards Subcommittee of the American Academy of Neurology. Neurology 2001;56:1143-53.

40. Mouton-Liger F, Wallon D, Troussière AC, Yatimi R, Dumurgier J, Magnin E, et al. Impact of cerebro-spinal fluid biomarkers of Alzheimer's disease in clinicalpractice: a multicentric study. J Neurol 2014;261:144-51. 
41. Beach TG, Monsell SE, Phillips LE, Kukull W. Accuracy of the clinical diagnosis of Alzheimer disease at National Institute on Aging Alzheimer Disease Centers,2005-2010. J Neuropathol Exp Neurol 2012;71:266-73.

42. Rinne JO, Brooks DJ, Rossor MN, Fox NC, Bullock R, Klunk WE, et al. 11C-PiB PET assessment of change in fibrillar amyloid-beta load in patients withAlzheimer's disease treated with bapineuzumab: a phase 2, double-blind,placebo-controlled, ascending-dose study. Lancet Neurol 2010;9:363-72.

43. Koolwijk I, Stein DS, Chan E, Powell C, Driscoll K, Barbaresi WJ. "Complex" attention-deficit hyperactivity disorder, more norm than exception? Diagnoses and comorbidities in a developmental clinic. J Dev Behav Pediatr 2014;35:591-7.

44. Nelson JM, Gregg N. Depression and anxiety among transitioning adolescents and college students with ADHD, dyslexia, or comorbid ADHD/dyslexia. J Atten Disord $2012 ; 16: 244-54$.

45. Caci HM, Bouchez J, Baylé FJ. An aid for diagnosing attention-deficit/hyperactivity disorder at adulthood: psychometric properties of the French versions of two Wender Utah Rating Scales (WURS-25 and WURS-K). Compr Psychiatry 2010;51:325-31.

46. Ramos-Quiroga JA, Nasillo V, Richarte V, Corrales M, Palma F, Ibáñez P, et al. Criteria and Concurrent Validity of DIVA 2.0: A Semi-Structured Diagnostic Interview for Adult ADHD. J Atten Disord 2019;23:1126-35.

47. Baron-Cohen S, Wheelwright S, Skinner R, Martin J, Clubley E. The autism-spectrum quotient (AQ): evidence from Asperger syndrome/high-functioning autism, males and females, scientists and mathematicians. J Autism Dev Disord. 2001;31:5-17.

48. Deb S, Hare M, Prior L, Bhaumik S. Dementia screening questionnaire for individuals with intellectual disabilities. Br J Psychiatry 2007;190:440-4. 
49. Scassellati C, Bonvicini C, Faraone SV, Gennarelli M. Biomarkers and attentiondeficit/hyperactivity disorder: a systematic review and meta-analyses. J Am Acad Child Adolesc Psychiatry 2012;51:1003-19.

50. Serrallach B, Groß C, Bernhofs V, Engelmann D, Benner J, Gündert N, et al. Neural Biomarkers for Dyslexia, ADHD, and ADD in the Auditory Cortex of Children. Front Neurosci 2016;10:324.

Figures et Légendes :

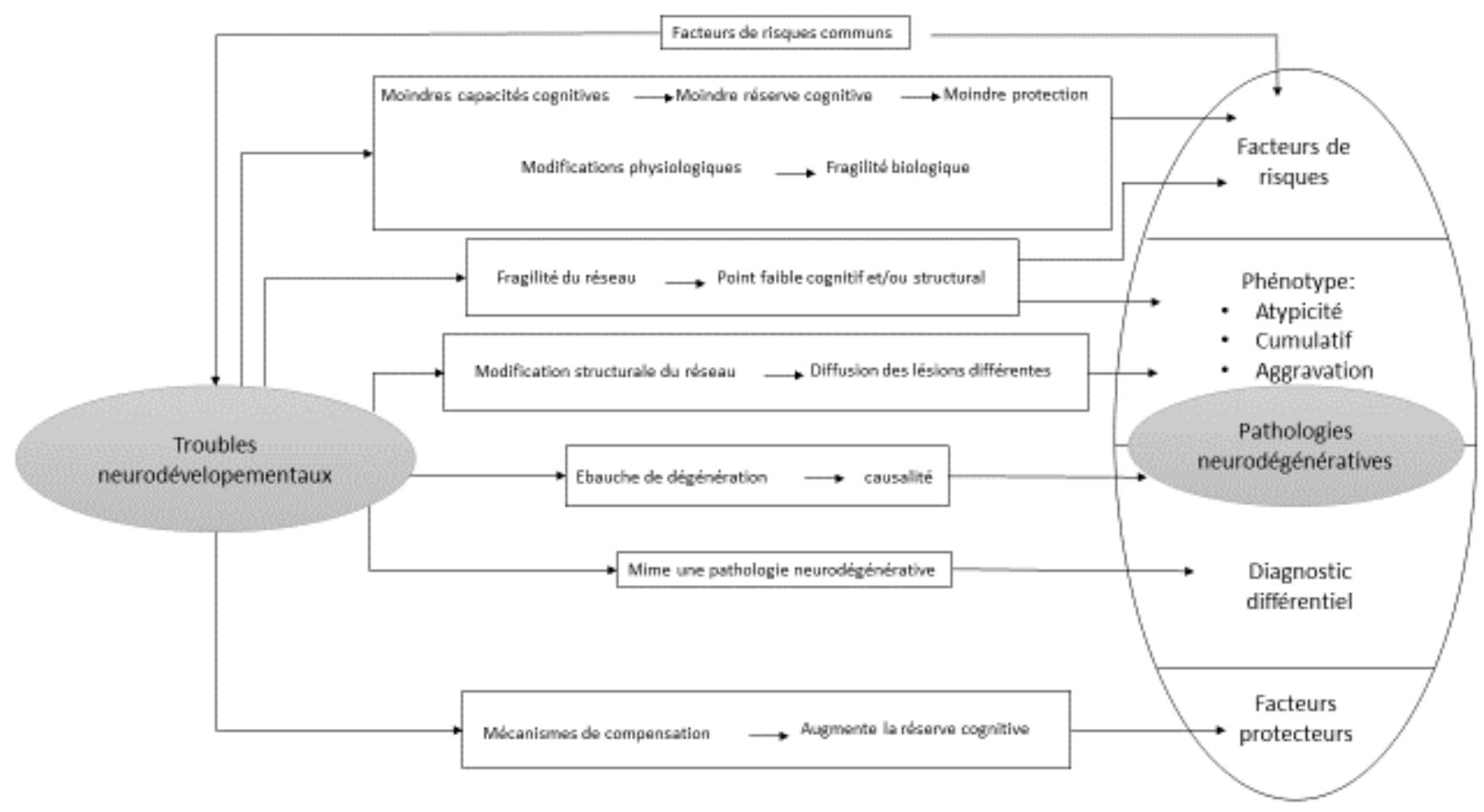

Figure 1. Quelques hypothèses d'interactions entre les troubles neurodéveloppementaux et neurodégénératifs qui ne sont pas mutuellement exclusives.

Figure 1. A selection of non-mutually exclusive hypotheses concerning the interactions between neurodevelopmental and neurodegenerative disorders. 


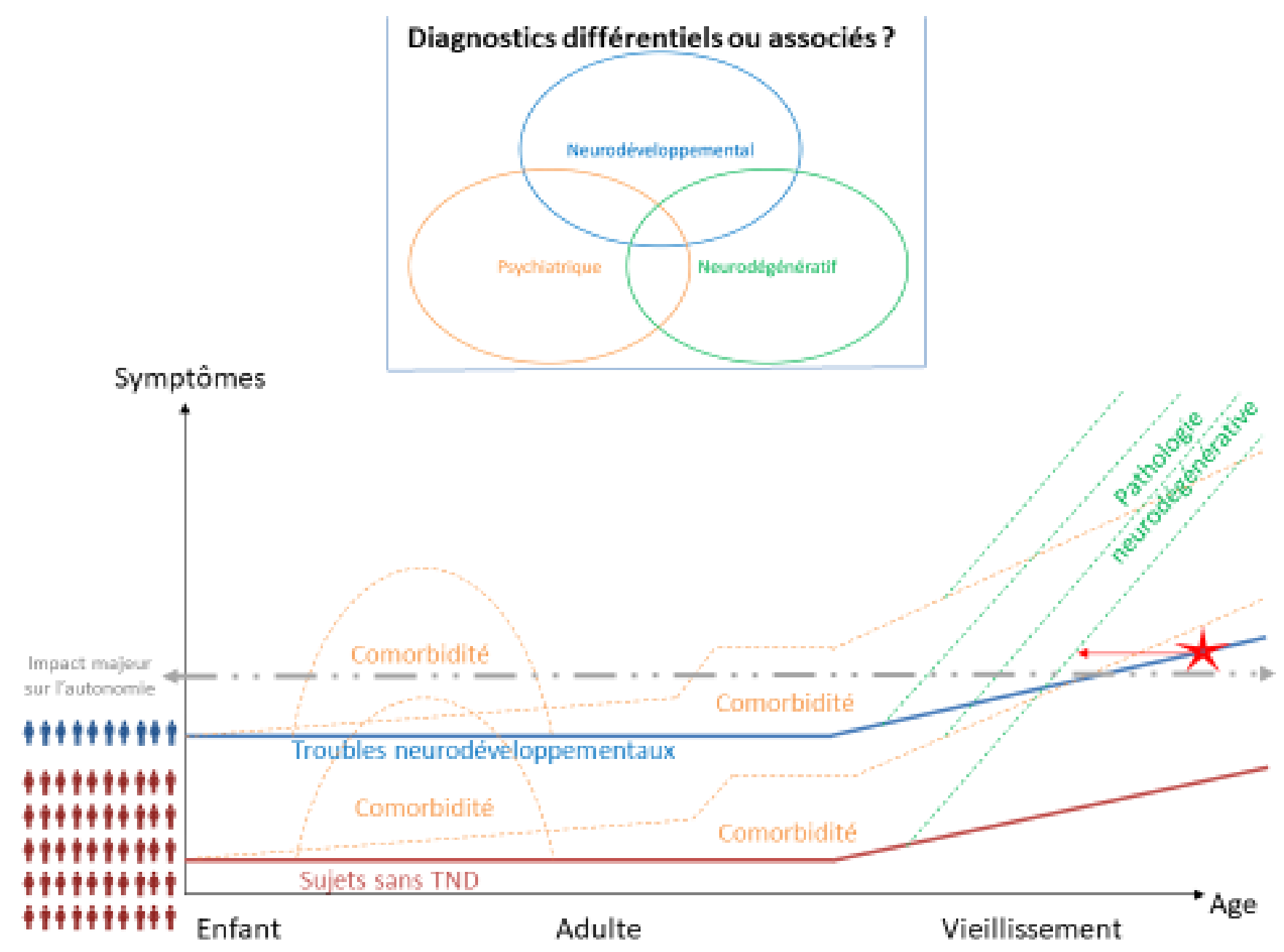

Figure 2 : Courbe représentant les symptômes des patients avec (bleu) et sans (marron) troubles neurodéveloppementaux (TND) tout au long de la vie et les intrications possibles entre les dimensions neurodéveloppementales, les comorbidités transitoires ou permanentes (psychiatriques, sommeil, somatiques, traumatismes crâniens, vasculaires, iatrogeniques, etc... représentées en orange) qui peuvent s'accumuler dans le temps, le vieillissement cognitif (pente de la courbe montrant l'accentuation des symptômes chez les plus âgés) et l'occurrence possible d'un authentique processus neurodégégrératif (vert). L'accumulation des symptômes peut ainsi entrainer un retentissement majeur sur l'autonomie (ligne pointillée grise) pouvant faire suspecter un trouble neurocognitif majeur. Les démarches diagnostiques sont ainsi complexes en terme de comorbidités, de facteur aggravant et/ou confondant et de 
diagnostics différentiels. L'étoile rouge cible un exemple de patient avec un TND vieillissant qui pourrait être considéré comme une maladie neurodégénérative si l'antécédent neurodéveloppemental n'est pas identifié, oublié ou sous-estimé (erreur diagnostic représentée par la flèche rouge).

Figure 2: Curve showing the lifetime trajectory of symptoms of patients with (blue) and without (brown) neurodevelopmental disorders. The graph depicts the possible interactions between the neurodevelopmental dimensions, temporary or permanent comorbidities (e.g. psychiatric, iatrogenic, sleep-related, somatic, brain trauma, vascular trauma, etc., (orange)), which can accumulate over time, cognitive aging (shown by the increased slope of the curves with aging), and the possible occurrence of a neurodegenerative disorder (green). The accumulation of symptoms can therefore have a significant effect on autonomy (dotted grey line), and together could be wrongly identified as a severe neurocognitive disorder. Diagnosis is therefore complicated because of comorbidities, aggravating/confounding factors, and differential diagnoses. The red star represents the example of a patient with an aging neurodevelopmental disorder, which could be wrongly diagnosed as a neurodegenerative disorder if the neurodevelopmental disorder is not identified. The neurodevelopmental disorder could also be forgotten about or underestimated (diagnostic error represented by the red arrow). 